Revista de Filosofía

Volumen 71 (2015) 23-30

\title{
ÉXITO PREDICTIVO Y REALISMO (CIENTÍFICO Y METAFÍSICO)
}

\author{
Manuel Comesaña \\ Universidad Nacional de Mar del Plata, Argentina \\ mcomesan@gmail.com
}

Resumen / Abstract

\begin{abstract}
El realismo científico enfrenta el problema de la subdeterminación de la teoría por los datos generada por la equivalencia empírica entre teorías rivales. Podría superarlo si fuera verdadero el predictivismo -el débil, ya que el fuerte tropieza con una dificultad que parece insuperable-. En tal caso, contribuiría además a refutar el escepticismo con respecto al mundo externo, pero no, como se ha sostenido, porque la experiencia dé más apoyo al realismo "ingenuo" del sentido común que a dicho escepticismo sino porque el realismo científico implica el realismo metafísico.
\end{abstract}

Palabras Clave: realismo científico, argumento del milagro, equivalencia empírica, predictivismo débil, realismo metafísico.

PREDICTIVE SUCCESS AND (SCIENTIFIC AND METAPHYSICAL) REALISM

Scientific realism faces the problem of the underdetermination of theory by evidence generated by the empirical equivalence of rival theories. It could overcome it if predictivism were true-weak predictivism, for strong predictivism stumbles over an apparently insurmountable difficulty. In that case, it would contribute also to the refutation of skepticism about the external world, but not, as it has been held, because experience supports more strongly the "naive" realism of common sense than such skepticism, but because scientific realism entails metaphysical realism.

KEY WORDS: scientific realism, miracle argument, empirical equivalence, weak predictivism, metaphysical realism.

R⿴囗十

En este trabajo se entiende por "realismo metafísico", como es habitual, la tesis de que hay un mundo fuera de nuestra mente que existe con independencia de ésta; y por "realismo científico"-rótulo que cubre varias tesis- la tesis de que las teorías (los sistemas de hipótesis teóricas) que han tenido un considerable éxito observacional deben ser verdaderas - parcialmente ${ }^{1}$ verdaderas, ya que la verdad total es improbable

1 No menciono las teorías aproximadamente verdaderas, como lo es la de Newton para ciertas velocidades, porque en sentido estricto esas teorías son totalmente falsas y no tienen 
en grado sumo-. El principal argumento a favor de esta tesis es que, de no ser así, dicho éxito sería inexplicable; sería un milagro, ha dicho Putnam².

Una fuente de dificultades para este "argumento del milagro" es el hecho de que todas las teorías tienen, o al menos pueden tener, rivales empíricamente equivalentes. Un caso particular de equivalencia empírica es el que se da entre una teoría más o menos verdadera y otra totalmente falsa, ejemplificado por la relación entre la teoría de Copérnico y una versión mejorada del sistema tolemaico. Si una teoría totalmente falsa puede tener las mismas consecuencias observacionales verdaderas que una teoría rival parcialmente verdadera, parecería prima facie que no se necesita verdad teórica para explicar el éxito de la ciencia.

Una manera de defender el argumento del milagro, y por lo tanto al realismo, frente a la tesis de la equivalencia empírica - muy probablemente la única maneraconsiste en elaborar la siguiente intuición: las teorías falsas que tienen consecuencias verdaderas, o bien no son totalmente falsas, esto es, son parcialmente verdaderas, o bien contienen hipótesis ad hoc; en este último caso su promesa de éxito observacional futuro es inferior a la de una teoría verdadera. La eficacia de esta estrategia depende de cuál sea el concepto de novedad predictiva que se esté manejando; si "El Sol va a salir mañana" se considera una predicción nueva, el realista está perdido: Tolomeo formula esa predicción tan bien como Copérnico. Es necesario proponer un concepto de predicción "novedosa", según el cual una teoría que solo mediante hipótesis ad hoc logra "salvar" fenómenos ya conocidos no es capaz de predecir otros que sean de distinto tipo en algún sentido razonablemente fuerte, y algunos autores lo han intentado 3 .

Esta estrategia realista supone admitir que una predicción exitosa tiene más fuerza confirmatoria que una explicación adecuada, esto es, supone tomar partido por la teoría histórica de la confirmación contra la teoría lógica, y con ello exige rechazar la idea de que el contexto de descubrimiento no puede influir en el contexto de justificación ${ }^{4}$. En efecto, que una consecuencia de una teoría sea algo que la teoría explica o algo que la teoría predice depende de la construcción o desarrollo de la teoría. Por ejemplo, la teoría de Torricelli explicó por qué es limitada la eficacia de las bombas y predijo que la presión atmosférica sostendría una columna de mercurio de aproximadamente setenta centímetros. La explicación tuvo cierto valor justificatorio -sobre todo porque ninguna otra teoría explicaba la limitación mencionada-, pero el éxito de la predicción lo tuvo en mayor grado. Como dice Popper, sin darse cuenta de que atenta contra la autonomía

consecuencias verdaderas -aunque sí consecuencias que son excelentes aproximaciones a la verdad, a tal punto que su diferencia con las verdades respectivas es tecnológicamente indetectable-.

"El realismo es la única filosofía que no hace del éxito de la ciencia un milagro" (1975, p. 73).

3 El esfuerzo más exhaustivo que conozco por explotar el concepto de predicción novedosa en beneficio del realismo científico es el de Leplin (1997).

$4 \quad$ Parece que el primero en señalar esto último fue Maher (1988). 
del contexto de justificación -que él defiende, aunque sin usar esa terminología-, el éxito predictivo garantiza que las premisas explicativas no son ad hoc.

Suelen negar la autonomía del contexto de justificación los partidarios de concepciones irracionalistas de la ciencia, pero no es eso lo que ocurre en este caso. El énfasis en el éxito predictivo va en contra de la distinción estándar entre descubrimiento y justificación de otra manera. La idea no es que cuán justificada esté una teoría depende de los procesos histórico-psicológicos que llevaron a su descubrimiento o invención, sino más bien que el éxito predictivo novedoso de la teoría varía inversamente con su adhocidad, cuestión que también es histórica ${ }^{5}$, pero en un sentido que no genera irracionalidad. Algunos sostienen que no se puede ser racionalista en la concepción de la ciencia sin ser también realista; si esto fuera cierto, parecería, contra lo que muchos han pensado, que una concepción racionalista de la ciencia está obligada a negar la autonomía de la justificación, y a asignarle un papel a la historia, como pedía Kuhn, aunque no el que él pedía.

Aun suponiendo resuelto el problema de elaborar un concepto satisfactorio de predicción novedosa, la estrategia realista que estoy examinando tropieza con una grave dificultad: no parece haber razones lógicas que justifiquen la preferencia por la predicción sobre la explicación -o "acomodación", como algunos la llaman-, ya que la subdeterminación de la teoría por los datos prima facie generada por la equivalencia empírica afecta a ambas por igual. Es sabido que cualquier conjunto de datos puede ser explicado por infinitas teorías distintas, de las cuales es verdadera una sola. Pero esto no es todo; si reemplazamos "explicado" por "predicho", la afirmación sigue siendo verdadera: cualquier conjunto de datos puede ser predicho por infinitas teorías distintas, de las cuales es verdadera una sola. No hay diferencias lógicas entre explicación y predicción; que la descripción de un hecho se deduzca de una teoría no tiene nada que ver con que ya se la conozca o todavía no, y éste es el principal argumento a favor de la teoría lógica de la confirmación, o "explicacionismo", como a veces se lo llama, y en contra de su rival, la teoría histórica, o "predictivismo". El problema para el predictivismo -y, por lo tanto, para el realismo- es entonces el siguiente: una teoría diseñada $a d$ hoc para explicar datos ya conocidos tiene la misma probabilidad de ser verdadera que una que tenga éxito predictivo; por lo tanto, la predicción no tiene intrínsecamente más valor confirmatorio que la mera acomodación. La tesis de que sí lo tiene se llama en la bibliografía sobre el tema "predictivismo fuerte".

Una posible manera de superar esta dificultad consiste en atribuirle importancia al éxito predictivo novedoso, no en sí mismo, sino como indicador de algún otro

5 Leplin (1997, pp. 68-77) no admite esto: él pretende reconstruir el razonamiento que condujo a una teoría sin adjudicar ningún papel a factores históricos contingentes. No voy a examinar aquí la cuestión de si esto es posible, aunque tengo la sospecha de que no hay razonamientos que conduzcan a teorías, y de que, para clasificar un resultado como novedoso con respecto a una teoría, no hay más remedio que exigir que no fuera conocido en el momento de formular la teoría-al menos, por el autor de la teoría-, por más que esto sea una circunstancia histórica contingente. 
rasgo asociado a las teorías y capaz de elevar su probabilidad. En esto consiste el "predictivismo débil". Así, se ha sostenido que dicho éxito indica: a) la posesión de un método confiable para generar teorías y predicciones (Maher 1988), b) que el teórico es "talentoso" (Kahn, Landsberg y Stockman 1992), c) que apunta de manera confiable a la verdad (White 2003), d) que la teoría no se ajusta demasiado a presuntos datos ya conocidos (es decir, no incurre en overfitting) (Sober y Hitchcock 2004), e) que son aproximadamente verdaderas las "creencias de fondo" de quien acepta la teoría (Barnes 2008), etc. ${ }^{6}$.

McCain (2012) considera al predictivismo débil como "incontrovertido" (uncontroversial) y sostiene que puede servir de base a un argumento en contra de las versiones tradicionales del escepticismo con respecto al mundo externo, esto es, en contra de la posibilidad de un genio maligno cartesiano y la de que seamos cerebros en una cubeta. Admite que no se lo puede alegar contra las versiones "mejoradas" de dicho escepticismo, pese a lo cual le parece de todos modos importante porque "proporciona un sólido argumento contra las hipótesis escépticas tal como son típicamente formuladas".

El argumento es el siguiente: las creencias de sentido común (SC), entre las cuales está la de que hay un mundo externo que no depende de nuestra mente, predicen, en la mayoría de los casos exitosamente, experiencias futuras, mientras que las hipótesis escépticas no lo hacen -lo único que pueden predecir es que el genio maligno nos va a seguir engañando, etc.-. Por lo tanto, dado el predictivismo débil, las experiencias sensoriales le dan un mayor grado de confirmación al SC que a las hipótesis escépticas, que explican o acomodan dichas experiencias pero no las predicen. McCain formaliza así el argumento:

1. El predictivismo débil es verdadero.

2. El SC predice exitosamente rasgos de las experiencias sensoriales que las hipótesis escépticas solo pueden acomodar.

3. No hay ningún rasgo de las experiencias sensoriales que las hipótesis escépticas predigan exitosamente y el SC, no.

4. Las experiencias sensoriales proporcionan apoyo indirecto a favor del SC que no proporcionan a favor de las hipótesis escépticas. (De 1), 2) 3))

5. Las experiencias sensoriales apoyan mejor al SC que a las hipótesis escépticas. (De 4))

6 Harker (2013) cita otras versiones del predictivismo débil, algunas de las cuales pueden ser descartadas porque la virtud que asocian al éxito predictivo no es capaz de elevar la probabilidad de una teoría. Es lo que ocurre con la de Lipton (2004), según la cual dicho éxito indica que el científico no ha manipulado (fudged) la teoría para ajustarla a los datos disponibles. Como se dijo en el texto, una teoría diseñada ad hoc para explicar datos ya conocidos tiene la misma probabilidad de ser verdadera que una que tenga éxito predictivo. 
A esta argumentación de McCain se le pueden hacer, y se le han hecho, objeciones. A continuación presento y discuto, simulando defender la postura de McCain, las que le hace Harker (2013), para después formular algunas propias. Por último, indico una vía alternativa para conseguir que el predictivismo débil dé apoyo al realismo metafísico.

1. Si el éxito predictivo no es valioso en sí mismo sino como indicador de alguna otra virtud de la teoría o del teórico, ¿por qué no tratamos de detectar directamente esa otra virtud? A lo mejor esto no es posible en el caso de las teorías científicas debido a que no sabemos lo suficiente, pero, ¿por qué no sería posible en el caso del sentido común? Los especialistas en la disciplina de que se trate sí podrían detectar directamente esa otra virtud: "Podríamos tener razones para creer que los expertos están en posesión de elementos de juicio (evidence) que nosotros ignoramos". Pero eso no ocurre en el caso del sentido común, que, como su nombre lo indica, todos compartimos.

La idea de que los científicos saben aquello que los filósofos de la ciencia están tratando de descubrir es extraña. Si valiera solo para este caso, la discusión del asunto por parte de filósofos de la ciencia no tendría sentido: bastaría con interrogar a los especialistas; si fuera generalizable, la filosofía de la ciencia no tendría derecho a existir. Cuando alguien sostiene que en efecto no lo tiene, el filósofo de la ciencia suele responder que es posible hacer algo sin saber, en algún sentido importante, qué es lo que se está haciendo, aun cuando se trate de una habilidad intelectual (nadie duda de que se pueda andar en bicicleta o nadar sin saber qué es lo que se está haciendo). Por ejemplo, al usar un lenguaje, aplicamos reglas de las que no somos conscientes y que no somos capaces de formular; podemos incluso equivocarnos si tratamos de formularlas, es decir, podemos equivocarnos al describir nuestro propio comportamiento lingüístico, y es perfectamente posible que ocurra algo análogo con las reglas del método científico, con las "creencias de fondo" de los científicos postuladas por Barnes, y con cualquier otra virtud que pueda asociarse al éxito predictivo. Einstein estaría de acuerdo, ya que, según cuentan, dijo: "Si usted quiere saber qué es la ciencia, lo peor que puede hacer es preguntarle a un científico". De modo que no es la falta de conocimientos científicos lo que impide la detección directa de la virtud asociada al éxito predictivo. Los científicos tampoco saben cuál es, y, como lo señala el propio Harker, entre los filósofos de la ciencia no hay siquiera consenso al respecto, mucho menos verdad probada. Y si los científicos pueden tener creencias inconscientes, ¿por qué no las demás personas? Suele hablarse, por ejemplo, de racismo inconsciente. No parece aventurado, en consecuencia, conjeturar que hay creencias inconscientes de sentido común que no pueden ser detectadas de manera directa sino solo a través del éxito predictivo.

2. En todas las versiones del predictivismo débil defendidas hasta ahora la virtud asociada al éxito predictivo se da solo en algunas situaciones, no en todas. 
Me parece dudoso que esto sea así respecto de la versión de Barnes (2008), que propone como virtud asociada al éxito predictivo las creencias "de fondo" de quien acepta la teoría, no las de quien la construye, aunque desde luego es posible, e incluso frecuente -pero no necesario, ya que no es imposible construir una teoría y considerarla falsa-, que una misma persona haga las dos cosas. Sostiene que esas creencias promueven la aceptación de la teoría, y que el éxito predictivo novedoso indica que son aproximadamente verdaderas. Por otra parte, si la virtud asociada al éxito predictivo se da en algunas situaciones, en esas situaciones el SC resulta mejor confirmado por la experiencia que las hipótesis escépticas, y no parece obvio que esto no brinde algún apoyo al argumento de McCain, ya que es al menos posible que no se den nunca las otras situaciones, esto es, aquellas en las que la predicción no tiene más fuerza confirmatoria que la acomodación. También existe la posibilidad de que la virtud en cuestión sea una todavía no descubierta y que se da en todos los casos. Harker desecha esta posibilidad con las siguientes palabras: "Por supuesto, es posible que McCain pueda concebir un nuevo predictivismo débil, que relacione el éxito predictivo con consideraciones que sean pertinentes para evaluar el SC y las hipótesis escépticas. Las tesis disponibles no están a la altura de la tarea”. No sé si tiene razón al no conceder ningún valor a versiones meramente posibles del predictivismo débil; de todos modos, creo que la respuesta a la siguiente objeción permite desechar también la que estamos discutiendo, al mostrar que el argumento puede prescindir de cualquier versión real o posible.

3. La parte incontrovertida del predictivismo débil es la que afirma que el éxito predictivo está asociado a alguna virtud de la teoría o del teórico, sin especificar cuál, como lo prueba el hecho de que haya unas cuantas versiones de dicho predictivismo, y ese "suelo compartido" no alcanza para justificar el argumento de McCain.

Sin embargo, si el éxito predictivo es tan reiterado que no puede ser atribuido al azar, debe estar asociado con alguna virtud de la teoría - de otro modo sería inexplicable; "sería un milagro"-, aunque no sepamos cuál es esa virtud, y esto es lo que ocurre con el sentido común, que acierta casi todo el tiempo. En consecuencia, lo que Harker reconoce como incontrovertido, a saber, el "suelo compartido" por todas las versiones particulares del predictivismo débil, basta para fundamentar el argumento de McCain.

Creo que es posible hacer a la propuesta de McCain objeciones más pertinentes que las formuladas por Harker, a saber, estas dos:

1. McCain pasa subrepticiamente de "incontrovertido" (uncontroversial) a "verdadero", lo que claramente es un non sequitur. Es más que obvio que el consenso - aun el consenso entre especialistas- no es garantía de verdad.

2. Las hipótesis escépticas no son empíricas porque son compatibles con cualquier experiencia y por lo tanto son irrefutables. Está mal, en consecuencia, decir -como lo hace McCain- que la experiencia sensorial da más apoyo al sentido común que al escepticismo, ya que a éste no puede darle ningún apoyo. Las predicciones del sentido común sí son refutables: a veces no sale 
agua cuando uno abre la canilla. Por lo tanto, el escepticismo con respecto al mundo externo no compite con el sentido común; al menos, no se trata de una competencia entre dos teorías empíricas, que pueda ser dirimida por el apoyo que las experiencias dan a cada una de ellas. El escepticismo con respecto al mundo externo es una teoría filosófica que no compite con el realismo "ingenuo" del sentido común sino con el realismo metafísico de los filósofos.

En realidad, el predictivismo contribuye a probar la existencia del mundo externo, pero no por la razón alegada por McCain, es decir, no porque la experiencia confirme más las creencias del SC que las hipótesis escépticas. Hay una vía distinta a través de la cual el predictivismo -el débil, ya que el fuerte enfrenta una dificultad que parece insuperable- puede apoyar al realismo metafísico. Como lo ha señalado Watkins (1984, pp. 349-350 y 1991, pp. 347-348), la exigencia de que la ciencia progrese a través de teorías cada vez más profundas presupone que existe una realidad externa y que es multinivelada; mejor dicho, que existe una realidad multinivelada y, por lo tanto, externa. Pero no solo esa exigencia sino también el realismo científico, al sostener que las teorías (los sistemas de hipótesis teóricas, el discurso científico sobre inobservables) que tienen éxito observacional deben ser parcialmente verdaderas, presupone una realidad multinivelada y por lo tanto externa: el mundo del solipsista tiene una sola capa. Por otro lado, si el predictivismo débil -en cualquiera de sus versiones, o aun en el "suelo compartido" por todas ellas- fuera verdadero, quedaría resuelto el problema de asignar una probabilidad más alta a las teorías que tienen éxito predictivo que a aquellas que solo acomodan los datos, y en consecuencia el "argumento del milagro" y con él el realismo científico no serían afectados por la subdeterminación de la teoría por los datos prima facie generada por la equivalencia empírica. En resumen: predictivismo débil $\rightarrow$ argumento del milagro $\rightarrow$ realismo científico $\rightarrow$ realismo metafísico. Se trata de un resultado condicional, ya que no está probado que el predictivismo débil sea verdadero, pero no carece de interés.

\section{Referencias bibliográficas}

Harker, D. W. (2013), "Discussion Note: McCain on Weak Predictivism and External World Scepticism", Philosophia 41: 195-202.

Kahn, J. A., S. E. Landsberg y A. C. Stockman (1992), "On Novel Confirmation", British Journal for the Philosophy of Science 43: 503-516.

Leplin, J. (1997), A Novel Defense of Scientific Realism. Nueva York: Oxford University Press.

Lipton, P. (2004), Inference to the Best Explanation. 2da. ed., Londres: Routledge.

Maher, P. (1988), "Prediction, Accommodation, and the Logic of Discovery", PSA 1988, Vol. I: 273-285. 
McCain, K. (2012), “A Predictivist Argument agains Scepticism”, Analysis 72: 660665.

Putnam, H. (1975), Mathematics, Matter and Method. Cambridge: Cambridge University Press.

Sober, E. y C. Hitchcock (2004), "Prediction Versus Acommodation and the Risk of Overfitting", British Journal for the Philosophy of Science 55: 1-34.

Watkins, John (1984), Science and Scepticism. Londres: Hutchinson.

(1991), "Scientific Rationality and the Problem of Induction: Responses to Criticism", British Journal for the Philosophy of Science 42: 343.

White, R. (2003), "The Epistemic Advantage of Prediction over Acommodation", Mind, 112-448: 653-683. 